\title{
Eficácia de um programa de exercícios físicos na qualidade de vida de mulheres com osteoporose
}

\author{
Efficacy of physical exercise program in life quality of women with osteoporosis
}

Marco Antonio Auad', Rodrigo Polaquini Simões', Sama Rouhani', Viviane Castello², Leda Shizuka Yogi

\section{Resumo}

Objetivos: Avaliar e comparar a qualidade de vida, por meio do Osteoporosis Assessment Questionnaire (OPA0), de mulheres com osteoporose, participantes e não participantes de um programa de atividade física, e comparar a qualidade de vida nas situações pré e pós-treinamento do grupo submetido à atividade física. Casuística e Métodos: Foram estudadas 28 mulheres com osteoporose, com idades entre 69 e 80 anos (76,1 $\pm 3,26$ anos) separadas em dois grupos: grupo 1 (G1) composto por 15 mulheres não participantes de um programa de atividade física (77,47 $\pm 2,26$ anos); e grupo 2 (G2) composto por 13 mulheres (74,46 $\pm 3,57$ anos) participantes de um programa de atividade física semanal. As participantes do G2 realizaram sessões de exercícios com duração de uma hora, duas vezes por semana, num período de oito meses. 0 questionário OPAQ foi aplicado com o objetivo de avaliar a qualidade de vida em ambos os grupos. 0 teste de Mann-Whitney foi utilizado para comparar os resultados entre os grupos, e 0 teste de Wilcoxon para comparar as situações pré e pós-treinamento do G2, e a avaliação inicial e final do G1. 0 nível de significância considerado foi de 5\%. Resultados: Verificou-se melhor pontuação em todos os domínios do G2 pós-treinamento (saúde geral, aspectos físicos, aspectos psicológicos, interação social, sintomas, dificuldades relacionadas ao trabalho e imagem corporal) tanto em relação a situação pré-treinamento como em relação ao G1. Conclusão: A prática de atividade física realizada regularmente pode representar importante instrumento na melhora da qualidade de vida de mulheres com osteoporose.

Unitermos: Osteoporose; qualidade de vida; atividade física.

\begin{abstract}
Objectives: To evaluate and compare the quality of life, by Osteoporosis Assessment Questionnaire (OPAO), of women with osteoporosis participating and not participating of a physical activity program and to compare the quality of life in pre and post training situations of group submitted to physical activity program. Subjects and Methods: A group of 28 women with osteoporosis aged 69-80 (76.1 \pm 3.26 years) was studied, being separated in two groups: group 1 (G1): 15 women who participated of a physical activity program (77.47 \pm 2.26 years); and group 2 (G2): 13 patients that participated in a physical activity program. The individuals from G2 did exercise sessions of one hour, twice a week, in a period of eight months. The OPAO was applied to evaluate the quality of life in both groups. The Mann-Whitney test was used to compare the results between the groups, and Wilcoxon test to compare the pre training situation with post training of G2, and the initial and final evaluations of G1. The significance level was $5 \%$ for all tests. Results: It was verified a better grading in all the domain of G2 post training situation (general health, physical aspects, psychological aspects, social interaction, symptoms, work related difficulties and body image), both on pre training situation of G2 as in relation to G1. Conclusion: The practice of physical activity done regularly can represent an important instrument on the improvement of quality of life, in women with osteoporosis.
\end{abstract}

Keywords: Osteoporosis; quality of life; physical activity.

Recebido: $23 / 11 / 2007$

Revisado: 05/03/2008

Aprovado: 16/04/2008

Universidade Federal de São Carlos (UFSCar), São Carlos (SP), Brasil

2 Centro Universitário Claretiano (CEUCLAR), Batatais (SP), Brasil

Faculdade de Medicina da Universidade de São Paulo (USP), São Paulo (SP), Brasi

Endereço para correspondência: Marco Antonio Auad, Rua Ana Prado, 526 - Vila Prado, CEP 13574-031 - São Carlos (SP). Tel.: (16) 3374-4112.

Email:ma auad@hotmail.com 


\section{Introdução}

Com o aumento da expectativa de vida da população brasileira e mundial, vários processos fisiológicos do organismo têm sua atuação reduzida devido ao processo de senescência, causando grande impacto na qualidade de vida, além de trazer prejuízos na realização das atividades de vida diária (AVDs), fazendo com que o grau de independência diminua. Dentre as várias alterações tem-se, com o avançar da idade, as alterações músculo-esqueléticas ${ }^{1}$, sendo a osteoporose considerada atualmente uma das doenças mais importantes que envolvem o sistema ósseo, além de sua grande incidência ${ }^{2}$.

A osteoporose é considerada uma doença sistêmica caracterizada por baixa massa óssea e deterioração microarquitetural do tecido ósseo, com conseqüente aumento da fragilidade óssea e susceptibilidade à fratura, segundo a Organização Mundial da Saúde $(\mathrm{OMS})^{2}$, e que atinge cerca de um terço das mulheres na pós-menopausa, sendo assim responsável por um alto índice de morbidade e mortalidade entre os idosos ${ }^{3-4}$.

O aparecimento da osteoporose está ligado à concentração dos hormônios do organismo. O estrógeno, um hormônio sexual feminino, também presente nos homens, mas em menor quantidade, ajuda a manter o equilíbrio entre a perda e o ganho de massa óssea. Nas mulheres, o ápice da massa óssea ocorre aproximadamente aos 35 anos de idade, e a partir desse período, gradativamente aumenta a atividade dos osteoclastos e diminui a atividade dos osteoblastos, resultando em perda de cálcio e massa óssea acentuada ${ }^{3}$. Além disso, há redução da força muscular e do processo sensório motor ${ }^{5}$, causando maior risco de quedas e fraturas, que atingem principalmente vértebras, extremidade distal do rádio (fratura de Colles) e colo do fêmur ${ }^{6-8}$. O risco de desenvolvimento da osteoporose no sexo feminino é quatro vezes maior do que em indivíduos do sexo masculino, sendo que 30\% das mulheres são acometidas no período de pós-menopausa, comprometendo as mesmas em diversos aspectos de sua vida?

Esses comprometimentos físicos provenientes da osteoporose podem ser responsáveis por comprometimentos emocionais, psicológicos e sociais, podendo limitar cada vez mais as atividades dos indivíduos idosos $^{3}$, causando diminuição da auto-estima e isolamento social ${ }^{8}$.

Com todas essas alterações físicas e emocionais torna-se cada vez mais necessário o desenvolvimento de tratamentos que visem reduzir as conseqüências trazidas pela osteoporose. A prática de atividade física melhora o estado de saúde física e psíquica de indivíduos de qualquer idade, inclusive de pacientes com osteoporose ${ }^{9}$. No estudo de Miszko ${ }^{10}$, avaliando mulheres no climatério e menopausa, observou-se que tanto os exercícios resistidos quanto os aeróbios melhoram a qualidade de vida e retardam muitas alterações fisiológicas relacionadas ao envelhecimento, amenizando o declínio da força muscular e da massa óssea, e conseqüentemente promovendo maior independência na realização das AVDs. Além disso, a prática de exercícios físicos regularmente proporciona maior bem estar mental, além de aumentar a deposição mineral óssea devido ao estresse mecânico causado pelos exercícios ${ }^{11-12}$.
Um dos métodos utilizados em larga escala para analisar a qualidade de vida de mulheres com osteoporose é a avaliação por meio dos questionários de qualidade de vida, já que estes apresentam baixo custo e fácil aplicabilidade. O Osteoporosis Assessment Questionnaire (OPAQ) é um questionário utilizado para avaliar a qualidade de vida de pacientes com osteoporose e possui versão validada para o português ${ }^{13}$. Para Rennó $^{14}$, o OPAQ é um instrumento adequado e de alta confiabilidade para avaliar a qualidade de vida de mulheres com osteoporose, já que faz uma abordagem global do indivíduo, informando a respeito tanto das limitações físicas, quanto dos comprometimentos emocionais e psicológicos. Diante disso, os objetivos deste estudo foram avaliar e comparar a qualidade de vida de mulheres com osteoporose participantes e não participantes de um programa de atividade física, tal como comparar a qualidade de vida nas situações pré e pós-treinamento do grupo submetido à atividade física, por meio do questionário OPAQ.

\section{Casuística e métodos}

\section{Voluntárias}

Foram estudadas 28 mulheres com osteoporose com idade entre $69 \mathrm{e}$ 80 anos (76,1 $\pm 3,26$ anos), encaminhadas pelo grupo de doenças ósseo-metabólicas do Hospital das Clínicas da Faculdade de Medicina da Universidade de São Paulo (HC/FMUSP). As voluntárias foram separadas em dois grupos de forma aleatória, a saber: grupo 1 (G1) composto por 15 mulheres não participantes de um programa de atividade física (77,47 $\pm 2,26$ anos), e grupo 2 (G2) composto por 13 mulheres (74,46 \pm 3,57 anos) participantes de um programa de atividade física semanal do Serviço de Fisioterapia do Instituto de Ortopedia e Traumatologia do HC/FMUSP.

Todas as voluntárias foram informadas sobre as características do estudo e orientadas em relação aos procedimentos que seriam realizados, e em seguida assinaram um termo de consentimento livre e esclarecido. O trabalho foi aprovado pelo comitê de ética em pesquisa com seres humanos da instituição, conforme determina a resolução 196/96 do Conselho Nacional de Saúde.

Foram selecionadas para participar deste estudo mulheres com faixa etária entre 60 e 80 anos, com diagnóstico clínico de osteoporose confirmada por raio-x e densitometria óssea, e aparentemente sem doenças neurológicas, musculares, articulares, cardiorrespiratórias e déficit cognitivo que as tornassem incapazes de participar do estudo.

\section{Avaliação e protocolo de exercícios}

Todas as mulheres foram submetidas à avaliação fisioterapêutica inicial, constituída de anamnese (coleta dos dados pessoais, história clínica atual e pregressa, e hábitos de vida), exame físico (inspeção física, força muscular, avaliação da amplitude de movimento articular, avaliação postural, ausculta cardíaca e pulmonar, dados antropométricos e mensuração da freqüência cardíaca - FC - e da pressão arterial - PA). 
Em seguida, somente o G2 iniciou um programa de atividade física, numa freqüência de duas vezes por semana, tendo duração de uma hora cada sessão, num período de oito meses. Inicialmente foram realizados dez minutos de alongamentos envolvendo tronco e membros, sendo cada alongamento mantido por 30 segundos no limite de cada voluntária.

O programa era composto por exercícios aeróbios leves como atividades com bastões e bola (envolvendo rotação, flexão e extensão de tronco; abdução, adução, flexão e extensão de ombros; e flexão e extensão de cotovelos e punhos) e exercícios resistidos de baixa carga (pesos livres de 0,5 a $3 \mathrm{~kg}$ ) envolvendo os membros superiores (os mesmos exercícios de ombro, cotovelo e punho realizados com bastão e bola) e inferiores (flexão, extensão, abdução e adução de quadril e flexão e extensão de joelhos, sendo os mesmos executados com caneleiras de 1 a $5 \mathrm{~kg}$ ). Todos os exercícios foram realizados em três séries de 12 repetições. A FC e a PA foram mensuradas antes e após a aplicação do protocolo de exercícios.

Antes da primeira sessão de exercícios, o grupo G2 foi submetido a um teste para determinação das cargas de cada tipo de exercício resistido, aos quais as pacientes seriam submetidas, sendo que cada voluntária deveria fazer 12 repetições com uma carga supostamente leve, e ao final era questionado o nível de esforço percebido utilizando-se a escala de Borg ${ }^{15}$ graduada de 0 a 10 pontos. A carga escolhida para iniciar o protocolo de exercícios era aquela em que a voluntária relatava esforço de intensidade leve (nível 2 da escala de Borg). O teste de carga foi realizado individualmente a cada duas semanas de treinamento, sendo a carga ajustada neste período.

\section{Avaliação da qualidade de vida}

O questionário OPAQ foi aplicado no G1 e G2 previamente ao treinamento físico. No G1 após oito meses da avaliação inicial e no G2 ao final do período de oito meses de treinamento, sendo realizado por um único avaliador, na forma de entrevista. As questões foram respondidas individualmente em uma sala fechada, para evitar possíveis influências de outras voluntárias nas respostas. Cada questão e suas alternativas foram lidas e explicadas pelo avaliador para que houvesse perfeito entendimento.

O OPAQ é um questionário composto por cinco perguntas gerais sobre saúde e qualidade de vida, e por 19 domínios compostos por perguntas relacionadas a 1. mobilidade (5 questões), 2. capacidade de andar e inclinar-se ( 5 questões), 3. dor nas costas (5 questões), 4. flexibilidade (4 questões), 5. cuidados próprios (4 questões), 6. tarefas de casa (4 questões), 7. movimentação (4 questões), 8. medo de quedas (5 questões), 9. atividade social (5 questões), 10. apoio da família e amigos (4 questões), 11. dor relacionada a osteoporose (5 questões), 12. sono (4 questões), 13. fadiga (4 questões), 14. trabalho (4 questões), 15. nível de tensão (5 questões), 16. humor (5 questões), 17. imagem corporal (4 questões), 18. independência (3 questões), e 19. atividade sexual (4 questões) $(14,15)$. As quatro questões relacionadas à atividade sexual (domínio 19) foram suprimidas, já que algumas participantes negaramse a respondê-las.

Para facilitar a análise do questionário, as cinco primeiras perguntas foram classificadas em uma única modalidade denominada saúde geral (SG). Os 18 domínios restantes foram agrupados em seis modalidades: aspectos físicos (AF) composto pelos domínios 1, 2, 4, 7 e 18; aspectos psicológicos (AP), domínios 15 e 16; interação social (IS) domínios 9 e 10; sintomas (ST) domínios 3, 8, 11, 12 e 13; trabalho (TB) domínios 5, 6 e 14; e imagem corporal (IC), composto pelo domínio 17 (16).

Cada pergunta dispõe de cinco respostas possíveis, pontuadas em uma escala de valores de $0,25,50,75$ e 100, sendo necessário que apenas uma seja escolhida - no caso, a que melhor represente as suas condições. O valor 100 foi atribuído às melhores condições e zero às piores, mas somente para as modalidades saúde geral e interação social; para as demais modalidades (aspectos físicos, aspectos psicológicos, sintomas, trabalho e imagem corporal) o zero representou as melhores condições e o 100 as piores.

\section{Análise estatística}

Para verificar a normalidade dos dados foi aplicado o teste de Shapiro-Wilk, e após constatada distribuição não normal, foi utilizado o teste não paramétrico de Mann-Whitney $\mathrm{U}$, a fim de comparar os valores das modalidades entre os grupos de idosas participantes e não participantes do programa de atividade física. Já o teste de Wilcoxon foi utilizado para comparar os valores pré e pós-treinamento do grupo submetido ao programa de exercício físico (G2) e a avaliação inicial e final do grupo não submetido ao treinamento físico. A probabilidade de ocorrência do erro do tipo I foi estabelecida em $5 \%$ para todos os testes $(\alpha=0,05)$. Os dados foram analisados por meio do programa Statistical Package for the Social Sciences $^{\mathrm{TM}}$ (SPSS - IBM, version 10.0.1, 1999).

\section{Resultados}

A Figura 1 ilustra os valores dos domínios avaliados por meio do questionário OPAQ: avaliação inicial e final (após oito meses) do G1 e do $G 2$ nas condições pré e pós-treinamento. Não foram observadas diferenças significativas quando comparados os valores do G1 (avaliação inicial) e G2 pré-treinamento, tal como entre a avaliação inicial e final do G1 Entretanto, foram verificados valores significativamente maiores nas modalidades SG e IS do G2 pós-treinamento em relação ao Gl (avaliação inicial e final). Já nas demais modalidades (AF, AP, ST, TB e IC) os valores apresentaram-se significativamente menores no G2 pós-treinamento.

Quando comparados os valores do G2 das situações pré e póstreinamento, foi verificado aumento significativo nas modalidades SG e IS e redução significativa nas modalidades AF, AP, TB e IC, na situação pós-treinamento (Figura 1). 


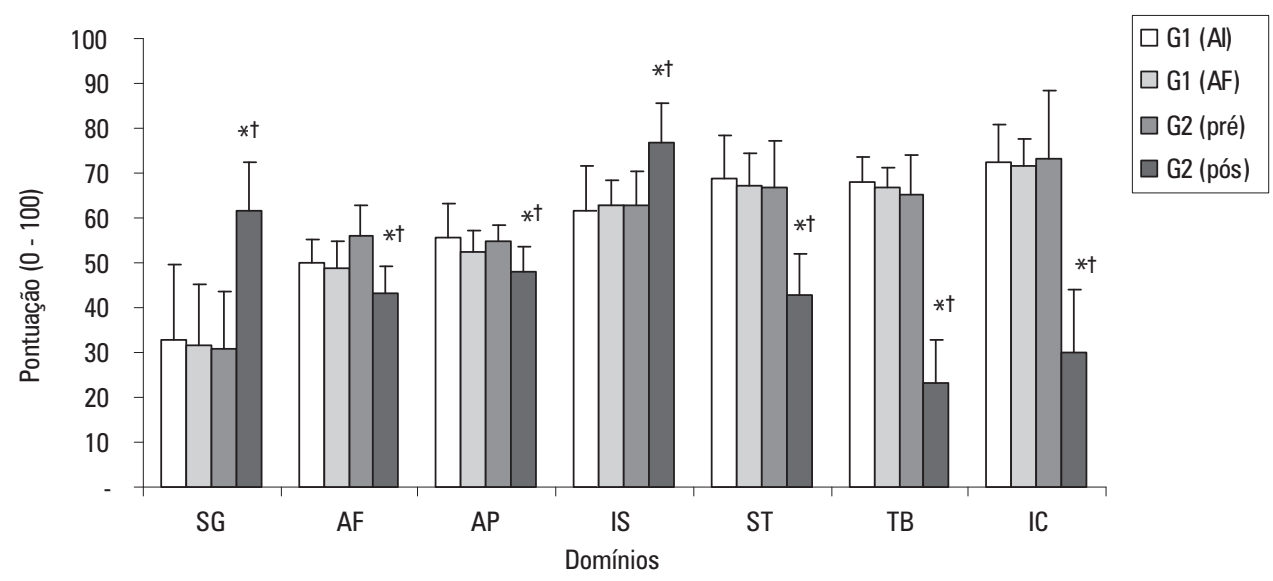

$\mathrm{SG}=$ saúde geral $/ \mathrm{AF}=$ aspectos físicos $/ \mathrm{AP}=$ aspectos psicológicos $/ \mathrm{IS}=$ interação social $/ \mathrm{ST}=$ sintomas $/ \mathrm{TB}=$ trabalho $/ \mathrm{IC}=$ imagem corporal *Diferença significativa $(\mathrm{p}<0,05)$ entre G1 (Al e AF) e G2 pós-treinamento.

tdiferença significativa $(p<0,05)$ entre G2 pré-treinamento e G2 pós-treinamento.

Figura 1 - Comparação dos valores em média e desvio padrão dos domínios avaliados por meio do questionário OPA0, entre os grupos de mulheres não praticantes de atividade física nas condições avaliação inicial (G1 Al) e avaliação final (G1 AF), e praticantes de atividade física nas condições de pré-treinamento (G2 pré) e pós-treinamento (G2 pós).

\section{Discussão}

Por meio dos resultados apresentados nesse estudo, foi possível verificar que o G1 (avaliação inicial) e o G2 na condição pré-treinamento, não apresentaram diferenças em relação à qualidade de vida, fato que comprova a homogeneidade dos grupos. No entanto, no pós-treinamento o G2 apresentou valores, em média, significantemente maiores, tanto em relação aos valores pré-treinamento, como em relação ao Gl (avaliação inicial e final) no que diz respeito à SG e IS, e valores significantemente menores nos domínios AF, AP, ST, TB e IC; resultados que representam melhores condições do G2 após o treinamento físico em relação à condição pré-treinamento, e em relação ao grupo que não realizou o protocolo de exercício físico (G1), demonstrando que o programa de atividade física pode proporcionar melhores condições em relação à qualidade de vida de mulheres com osteoporose.

Em relação às condições de saúde geral, o G2 apresentou maiores valores na situação pós-treinamento quando comparada tanto à condição de pré-treinamento como em relação ao G1, sendo que esses resultados corroboram o estudo de Bloomfield ${ }^{16}$, que encontrou uma relação benéfica entre a prática de atividade física e a melhora das condições de saúde geral. Neste sentido, esses resultados ressaltam a importância da implementação de exercícios físicos para a população idosa, principalmente quando a osteoporose está presente.

As participantes do programa de atividade física apresentaram ao final do treinamento melhor desempenho nos aspectos físicos em relação à condição pré-treinamento, bem como em relação ao grupo não treinado. Esses resultados sugerem que após oito meses de treinamento, as idosas apresentaram maior facilidade em relação à mobilidade corporal, proporcionando maior nível de independência em relação às AVDs, concordando com o estudo de Matsudo et al. ${ }^{11}$. Aveiro et al. ${ }^{17}$, aplicaram 12 semanas de treinamento físico em 16 mulheres com osteoporose visando melhora no equilíbrio e na força muscular do quadríceps destas voluntárias. Os autores ${ }^{17}$ verificaram melhora significativa nas AVDs devido à melhora do torque dos músculos extensores do quadríceps, e conseqüentemente do equilíbrio e da confiança destas idosas, proporcionando melhora na qualidade de vida desta população.

Em nosso estudo o G2 apresentou ao final do treinamento físico menor valor em relação aos aspectos psicológicos, o que demonstra influência benéfica da prática de exercícios à saúde mental tanto em relação à condição pré-treinamento, como em relação às não praticantes de atividade física, corroborando os estudos de Hauer et al. ${ }^{18} \mathrm{e}$ Driusso et al..$^{19}$, que observaram melhora emocional e comportamental como conseqüência de um programa de atividade física para pacientes geriátricos frágeis.

Quanto à interação social, nossos resultados sugerem maior sociabilidade do grupo praticante de atividade física ao final do treinamento, como também em relação ao grupo não praticante de atividade física. Esses achados podem estar relacionados à limitação física encontrada no G1, já que Kowalski et al..$^{20}$, verificaram forte relação entre as limitações físicas e o isolamento social em mulheres osteoporóticas. Além disso, a maior interação social verificada nas praticantes de atividade física pode estar relacionada ao fato destas mulheres terem realizado os exercícios em grupo, e poderem compartilhar suas experiências de vida, o que pode ter interferido positivamente neste processo ${ }^{19}$.

No que diz respeito aos sintomas, o G2 apresentou ao final do treinamento menores valores relacionados à dor quando comparados à situação pré-treinamento e ao G1 (avaliação inicial e final). Os alongamentos aplicados antes do protocolo de exercícios aeróbios e resistidos localizados, talvez tenham contribuído para redução da tensão muscular e conseqüentemente para o alívio da dor. Esses resultados são concordantes com outros estudos prévios que verificaram diminuição do nível de dor, tanto após o treinamento com exercícios aeróbios ${ }^{21}$ como após exercícios para fortalecimento muscular ${ }^{17}$. 
Quanto ao domínio trabalho, o grupo de participantes do programa de atividade física se beneficiou com o protocolo proposto, pois seus valores foram menores em relação à situação pré-treinamento e ao G1 (avaliação inicial e final), o que traduz em melhores condições. Provavelmente a prática da atividade física tenha promovido efeitos benéficos em relação à facilidade de realizar as $\mathrm{AVDs}^{22}$, fato que pode estar associado à melhora de outros domínios como: interação social, sintomas, aspectos físicos e psicológicos verificados no grupo praticante de atividade física.

Em relação à imagem corporal, o G2 após o treinamento de oito meses, declarou ter observado menor presença de deformidades corporais conseqüentes da osteoporose quando comparada à situação prétreinamento, e quando o mesmo foi comparado ao G1 (avaliação inicial e final), apresentou menor valor, o que representa melhores condições em relação a esse domínio. Estes resultados podem estar relacionados com a melhora dos fatores físicos observados no grupo praticante de atividade física, como por exemplo, a melhora da flexibilidade, da capacidade de inclinar-se e de movimentação em geral, que tem forte relação com os aspectos psicológicos e estes, consequentemente com a imagem corporal ${ }^{23}$.

Em vista dos resultados obtidos neste estudo, podemos concluir que mulheres osteoporóticas submetidas a um programa de atividade física, com duração de oito meses, apresentaram melhor qualidade de vida ao final do treinamento físico, tal como quando comparadas a mulheres osteoporóticas não participantes de um programa de atividade física.

\section{Referências}

1. Perracine MR, Ramos LR. Fatores associados a quedas em uma coorte de idosos residentes na comunidade. Rev Saúde Pública 2002;36(6):709-16.

2. World Health Organization. Assessment of fracture risk and its application to screening for posmenopausal osteoporosis: report of a WHO study group. Technical Report Series 1994;843.

3. Johnell, 0. Advances in osteoporosis: better identification of risk factors can reduce morbidity and mortality. J Int Med 1996;239(4):299-304.

4. Kannus P, Niemi S, Parkari J, Palvaren N, Vuor J, Jyarvinen J. Hip fractures in Finland between 1970 and 1997 and predictions for the future. Lancet 1999;353(9155):802-5.

5. Lynn SG, Sinaki M, Westerlind KC. Balance characteristics of persons with osteoporosis. Arch Phys Med Rehabil 1997;78(3):273-7.

6. Galsworthy TD, Wilson PL. Osteoporosis. It steals more than bone. Am J Nurs 1996;96(6):26-33.

7. Oleksik AM, Ewing S, Shen W, van Schoor NM, Lips P. Impact of incident vertebral fractures on health related quality of life (HROOL) in postmenopausal women with prevalent vertebral fractures. Osteoporos Int 2005;16(8):861-70.

8. Gold DT. The clinical impact of vertebral fractures: quality of life in women with osteoporosis. Bone 1996;18(3 suppl):185S-9.

9. Rebelatto JR, Calvo JL, Orejuela JR, Portillo JC. Influência de um programa de atividade física de longa duração sobre a força muscular manual e a flexibilidade corporal de mulheres idosas. Rev Bras Fisioter 2006;10(1):127-32.

10. Miszko TA, Cress ME. A lifetime of fitness. Exercise in the perimenopausal and postmenopausal woman. Clin Sports Med 2000;19(2):215-32.

11. Matsudo SMM, Matsudo VKR. Osteoporose e atividade física. Rev Bras Cienc Mov 1991;5(3):33-59.

12. Meeks SMPT. The role of the physical therapist in the recognition, assessment, and exercise intervention in persons with, or at risk for, osteoporosis. Top Geriatr Rehabil 2005;21(1):42-50.
13. Cantarelli FB, Simões MFJ, Oliveira LM, Ferraz MB, Szejnfeld VL. Qualidade de vida em pacientes com fraturas por osteoporose: adaptação cultural, reprodutibilidade e validação do Osteoporosis Assessment Questionnaire. Rev Bras Reumatol 1999;39(1):9-18.

14. Rennó ACM. Efeitos de um programa de exercícios no grau de cifose torácica, na função pulmonar e na qualidade de vida de mulheres com osteoporose. Tese. Universidade Federal de São Carlos. São Paulo: 2003, p.90-5.

15. Borg, GA. Psychophysical bases of perceived exertion. Med Sci Sports Exerc 1982;14(5):377-81

16. Bloomfield SA. Contributions of physical activity to bone health over the lifespan. Top Geriatr Rehabil 2005;21(1):68-76

17. Aveiro MC, Navega MT, Granito RN, Rennó ACM, Oishi J. Efeitos de um programa de atividade física no equilíbrio e na força muscular do quadríceps em mulheres osteoporóticas visando uma melhoria na qualidade de vida. Rev Bras Cienc Mov 2004;12(3):33-8.

18. Hauer K, Rost B, Rutschle K, Opitz H, Specht N, Bartsch P, et al. Exercise training for rehabilitation and secondary prevention of falls in geriatric patients with a history of injurious falls. JAGS 2001;49:10-20.

19. Driusso P, Oishi J, Rennó ACM, Ferreira V. Efeitos de um programa de atividade física na qualidade de vida de mulheres com osteoporose. Rev Fisioter Univ São Paulo 2000;7:1-9.

20. Kowalski SC, Sjenzfeld VL, Ferraz MB. Resource utilization and costs in osteoporosis. Rev Assoc Med Bras 2001;47(4):352-7.

21. Navega MT, Oishi J. Comparação da qualidade de vida relacionada à saúde entre mulheres na pós-menopausa praticantes de atividade física com e sem osteoporose. Rev Bras Reumatol 2007;47(4):258-64.

22. Williams GN, Higgins MJ, Lewek MD. Aging skeletal muscle: physiologic changes and the effects of training. Phys Ther 2002;82(1):62-8.

23. Lemos MCD, Miyamoto, ST, Valim V, Natour J. Qualidade de vida em pacientes com osteoporose: correlação entre OPAO e SF-36. Rev Bras Reumatol 2006:46(5):323-8. 